\title{
Forecasting future dental treatment needs
}

\section{Patient history as a predictor of future treatment need? Considerations from a dataset containing over nine}

million courses of treatment. Br Dent J 2020; 228: 345-350. https://doi.org/10.1038/s41415-020-1305-4

Humans have always been fascinated with predicting the future. The only difference between the past and the present is the methods we use to make these predictions. Long gone are the days of oracles and palm readers, they have now been replaced with sophisticated mathematical models and technology, such as those seen in weather forecasting and actuarial science.

In dentistry, one example where forecasting is applied is in the prediction of future dental treatment need, which: (a) helps health service providers anticipate the population's treatment needs and plan funding; (b) advises insurance companies on the setting of premiums; and (c) aids clinicians in their business planning and tailoring of recall intervals and preventive advice to patients. A patient's treatment history is commonly accepted to be a good predictor of their future treatment need, but there is actually little evidence in the literature to support this claim. Therefore, the aim of this study was to determine whether overall patient treatment history could predict future treatment need.

To do this, the authors analysed a dataset containing the treatment records of adult patients treated in the General Dental Services (GDS) of England and Wales between 1990 and 2006. A total of 455,844 patients and 9,341,583 courses of treatment (CoT) were included in the study. Of these, $49 \%$ were 'active' CoTs (eg,

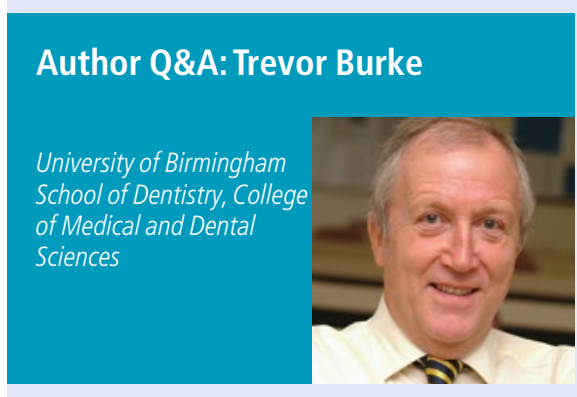

Why did you decide to undertake this study?

We undertook the study because we have the benefit of one of the largest longitudinal datasets in the world relating to dental treatment, and, while the perceived wisdom was that future patient treatment was related to past treatment history, we decided to ascertain if this was correct. extractions) while $51 \%$ were 'non-active' CoTs (eg, prevention and diagnosis).

Overall, the study revealed a positive correlation between treatment history and future treatment need. This correlation was even stronger with active CoTs in particular. In other words, patients who had more active treatment in the past are more likely to need active treatment in the future. In contrast, there was a negative correlation between historical non-active treatment costs and future active treatment costs. This means that patients who had more non-active treatment (for example, prevention) in the past are less likely to need active treatment in the future; a finding which is congruent with current concepts of prevention-focused treatment planning.

The authors also found that the prediction accuracy improved with a larger dataset of treatment history. However, data after 5 years did not significantly improve the correlation. In fact, even just one year of treatment history data could provide a reasonable estimate of future treatment need at the Heath Authority level, which means forecasting could be done reliably at low cost. This work provides a proof of concept that if further developed, could produce an operational predictive model for practices and the NHS.

Jed Y. J. Lee

Guy's and St Thomas' NHS Foundation Trust)

\section{Did any of the results surprise you?}

Yes, the surprise was that only relatively short time periods of historical data were needed in order to project future treatment need. Although the more data the better, good results could be expected using only one or two years of history.

\section{What do you think the next steps should be} considering your findings?

The next steps should include developing predictive models at practice or health authority level. For compatibility with current recording requirements, these could be based on Units of Dental Activity rather than scale fees, provided the data on UDA are available. 